\title{
FUZZY GOAL PROGRAMMING APPROACH ON COMPUTATION OF THE FUZZY ARITHMETIC MEAN
}

\author{
Mustafa Güneş and Nurullah Umarosman \\ Univ. of EMU, Department of Industrial \\ Engineering, Gazi Magusa, TRNC (via Mersin 10,Turkey) \\ mustafa.gunes@emu.edu.tr \\ Univ.of Dokuz Eylul, Fac. of Economic \& Adm.Sciences, Dept. of Econometrics \\ Buca, Izmir, Turkey
}

\begin{abstract}
In the traditional way of computing the arithmetic mean, there are various alternatives that support the same objective. Since the proposal of fuzzy sets by Zadeh, Bellman and Zadeh have developed a basic framework for decision-making in a fuzzy environment and investigated the fuzzy c-mean and the fuzzy weighted averages. The fuzzy c-mean is an extension of the c-mean that is widely applied to cluster algorithm. The fuzzy weighted averages extend the normal weighted mean by using the concept of fuzzy numbers and the extension principle. The fuzzy arithmetic mean is an extension of the normal arithmetic mean.

The Goal Programming, which is used to solve the multiple objective decision problems, has wide and great potential among other methods targeting maximization or minimization of goals. The goal programming aims to minimize the biases from each objective, instead of optimizing of goals.

In this study we do introduce a new approach to the computation of fuzzy arithmetic mean powered by fuzzy goal programming, named GUMAR.
\end{abstract}

Keywords - Fuzzy Arithmetic mean, Goal programming, Fuzzy Goal Programming.

\section{1-INTRODUCTION}

One of the important objectives of the statistical study is to estimate the parameters of population that determine the shape of the distribution of the data that are gathered from a research field. The arithmetic mean and variance values of the gathered data form a research field are essential information to improve additional inference about the basis of populations. There are various alternative ways for computing the arithmetic mean that supports the same target. Since the proposal of the concept of fuzzy sets by Zadeh, Bellman and Zadeh have developed a basic framework for decision-making in a fuzzy environment [1] and then the fuzzy c-mean and the fuzzy weighted averages have been investigated. The fuzzy c-mean is an extension of the cmean that widely applied to cluster algorithm[2].The fuzzy weighted averages extends the normal weighted mean by using the concept of fuzzy numbers and the extension principle[3]. As an alternative approach, The Goal Programming (GP) Technique is a very useful tool for decision makers to discuss and find a set of suitable and acceptable solutions to decision problems. Many decision problems of top managers have been solved so far. However, determining precisely the goal value of each objective is difficult for decision maker, because only partial information can be obtained.

Thereafter, many researches followed in which Narasimhan and Hannan have extended the fuzzy set theory to the field of goal programming. In this study, the fuzzy 
goal programming approach is used to determine the Fuzzy Arithmetic Mean as a first typical application. In fact, the fuzzy goal and multi -objective programming has very wide spread application.

For example, Per lack and Willis considered the application of a multi-objective programming model in a sludge disposal problem in the USA. Koo et al. (1991) accomplished the sitting planning of a regional hazardous waste treatment center by using a fuzzy multi-objective programming technique in Korea [4], and Fuzzy Goal Programming Approach for Water Quality Management in a River Basin has published in Fuzzy Sets and Systems [5]. M. Arenas Parra, A. Bilbao Terol, M.V. Rodriguez Uria have discussed a Fuzzy Goal Programming Approach to Portfolio Selection [6], LiangHsuan Chen, Feng-Chou Tsai have formulated fuzzy goal programming (FGP) incorporating different importance and preemptive priorities by using an additive model to maximize the sum of achievement degrees of all fuzzy goals [7]. T. K. Roy and M. Maiti have discussed Multi-Objective inventory models of deteriorating items with some constraints in fuzzy environment [8] and Jong Soon Kim and Kyu-Seung Whang have investigated the application of tolerance concepts to goal programming in a fuzzy environment [9].

Three methodologies capable of effectively dealing with multi-objective programming problems are vector maximum (VM) methods, goal programming (GP) approaches and interactive techniques [5]. Every approach has its own advantages and disadvantages depending on their structures. Briefly, VM method has the advantages of variety of alternatives it yields. GP approaches have directly arrived at an acceptable compromise solution. The interactive techniques are more often desirable because they yield a single preferred solution. A disadvantage of those three methods is their strong dependence upon local information that occasionally cannot arrive at an "optimal" solution.

There is a quite difference between Goal Programming and Fuzzy Goal Programming such as follows; Goal Programming requires the DM to set definite aspiration values for each objective that he/she wishes to achieve, whereas the latter is specified in an imprecise manner. A fuzzy goal is considered here as a goal with an imprecise aspiration level. Consideration of different relative importance and priorities of the goals in the Fuzzy Goal are proper than others. Narasimhan has used linguistic variables, such as "very important", "less important" and "moderately important", to describe the fuzzy weights of the goals, and defined the corresponding membership functions by specifying the desirable intervals of membership degree to reflect the importance [10]. In this study we do introduce new approach of computation of fuzzy arithmetic mean, named by us, GUMAR. Before introducing the GUMAR and its numeric application, recalling the alternative models of FGP could be more significant to guide the flow of discussions.

\section{2- MODELS OF THE FUZZY GOAL PROGRAMMING}

Programming is important because some of the goals are less or more important A classical structure of the multi-objective programming model is as follows.

$\operatorname{Max} A \mathbf{x}$

$$
\text { s.t. } \mathrm{Cx} \leq \mathrm{d} \text {, }
$$


$\mathrm{x} \geq 0$

Where $\mathrm{x}$ is an (nx1) alternative set, $\mathrm{A}$ is an (mxn) matrix of coefficients of objective functions, $C$ is a (pxn) matrix of coefficients of constraints and $d$ is a (px1) right-hand side values of model.

The model (2.1) can be reformulated as a Fuzzy Goal Programming problem, in the case of presentation of fuzzy information in which the aspiration level set $b_{0}$ can be constructed by using the pay-off table, such as below;

\section{Find $x$}

$$
\begin{array}{ll}
\text { s.t. } & \mathrm{Ax} \cong \mathrm{b}_{0}(\text { or } \tilde{b}) \\
& \mathrm{Cx} \leq \mathrm{d}, \\
& \mathrm{x} \geq 0,
\end{array}
$$

where both the symbol $\cong($ or $\tilde{b})$, express linguistic goals such as "the profit should be around b".

In addition to above alternatives, if it is possible to consider the RHS values as a fuzzy(fuzzy resources), the model (2.2) can be written as

\section{Find $x$}

$$
\begin{array}{ll}
\text { s.t. } & \mathrm{A} \mathrm{x} \cong \mathrm{b}_{0}(\text { or } \widetilde{b}) \\
& \mathrm{C} \mathrm{x} \\
& \cong \mathrm{d}, \\
\mathrm{x} \geq 0 &
\end{array}
$$

where the fuzzy equality constraints express that earned profits should be around $b_{i}$, and the symbol $\cong$ indicates the fuzziness of the constraint and is read as "approximately less than or equal to". The equation (2.2) and (2.3) can be solved by similar methods if similar membership functions are used for modeling the imprecise nature of "fuzzy goals" and "fuzzy equality".

To resolve these fuzzy equalities, we should elicit their membership functions based on a preference concept from the Decision Maker(s). For the sake of the mathematical tractability, the membership function $\mu_{i}(x)$

$$
\mu_{i}(x)=\left\{\begin{array}{cc}
{\left[(A x)_{i}-\left(b_{i}-d_{i}\right)\right] / d_{i}} & b_{i}-d_{i} \leq(A x)_{i}<b_{i} \\
{\left[\left(b_{i}-d_{i}\right)-(A x)_{i}\right] / d_{i}} & b_{i} \leq(A x)_{i} \leq b_{i}+d_{i} \\
0 & b_{i}+d_{i}<(A x)_{i} \text { or }(A x)_{i}<b_{i}-d_{i}
\end{array}\right.
$$

To solve Equation (2.3), when Equation (2.4) is given, NARASIMHAN proposed the following $2^{m}$ sub-problems of equivalent standard linear goal programming:

$$
\begin{aligned}
\max \left\{\min _{i}\left[(A x)_{i}-\left(b_{i}-d_{i}\right)\right] / d_{i}\right\} & \\
\text { such that } & b_{i}-d_{i} \leq(A x)_{i} \leq b_{i} \\
x & \geq 0
\end{aligned}
$$


and

$$
\begin{gathered}
\max \left\{\min _{i}\left[\left(b_{i}-d_{i}\right)-(A x)_{i}\right] / d_{i}\right\} \\
\text { such that } \quad b_{i} \leq(A x)_{i} \leq b_{i}+d_{i} \\
x \geq 0
\end{gathered}
$$

By putting equations (2.5) and (2.6) together, we obtain

$$
\begin{array}{ll}
\max & \alpha \\
\text { s.t } & {\left[(A x)_{i}-\left(b_{i}-d_{i}\right)\right] / d_{i} \geq \alpha} \\
& b_{i}-d_{i} \leq(A x)_{i} \leq b_{i} \\
& {\left[\left(b_{i}-d_{i}\right)-(A x)_{i}\right] / d_{i} \geq \alpha} \\
& b_{i} \leq(A x)_{i} \leq b_{i}+d_{i} \\
& \alpha \in[0,1] \text { ve } x \geq 0
\end{array}
$$

Moreover, the first two constraints of equation (2.7) can be expressed as

$\alpha \leq 1+\left[(A x)_{i}-b_{i}\right] / d_{i}$ and $\left(b_{i}-d_{i)} / d_{i} \leq(A x)_{i} / d_{i} \leq b_{i} / d_{i}\right.$

let us $(A x)_{i} / d_{i}=b_{i} / d_{i}-\delta_{i}^{+}$, where $\delta^{+}$is overestimated; and we have;

$\alpha+\delta_{i}^{+} \leq 1$ ve $(A x)_{i} / d_{i}-\delta_{i}^{+}=b_{i} / d_{i}$

Similarly, third and forth constraints of Equation (2.7) are equivalent to:

$\alpha+\delta_{i}^{+} \leq 1$ and $(A x)_{i} / d_{i}-\delta_{i}^{+}=b_{i} / d_{i}$

Hannan then combined Equation (2.9) and (2.10) to obtain the following linear goal programming model which is equivalent to NARASIMHAN $2^{m}$ sub- problems:

$$
\begin{array}{cl}
\max & \alpha \\
\mathrm{s.t} & (A x)_{i} / d_{i}+\delta_{i}^{-}-\delta_{i}^{+}=b_{i} / d_{i} \\
& \alpha+\delta_{i}^{-}+\delta_{i}^{+} \leq 1 \\
& \delta_{i}^{-}, \delta_{i}^{+} \geq 0 \\
& \delta_{i}^{-} \delta_{i}^{+}=0 \\
& \alpha \in[0,1] \text { and } x \geq 0
\end{array}
$$

on the other hand, Yang, İgnizio, and Kim used Zimmermann's fuzzy programming to solve (2.2) with membership functions of (2.4) and obtained the following auxiliary model:

$$
\begin{aligned}
& \max \alpha \\
& \text { s.t. }\left[(A x)_{i}-\left(b_{i}-d_{i}\right)\right] / d_{i} \geq \alpha \\
& {\left[\left(b_{i}+d_{i}\right)-(A x)_{i}\right] / d_{i} \geq \alpha} \\
& \alpha \in[0,1]
\end{aligned}
$$

if the deviations $\mathrm{d}_{i}$ from the centers $b_{i}$ are different, we would have the following model:

$$
\begin{array}{ll}
\max \alpha & \\
\text { s.t. } & {\left[(A x)_{i}-\left(b_{i}-d_{i 1}\right)\right] / d_{i 1} \geq \alpha} \\
& {\left[\left(b_{i}+d_{i} 2\right)-(A x)_{i}\right] / d_{i 2} \geq \alpha}
\end{array}
$$


Comparison of equation (2.7), (2.11) and (2.12) with isosceles triangular membership function are given below:

\begin{tabular}{lccc} 
Equation & no. Of LP & no. Of Constraints & no, of Variables \\
\hline 2.7 & $2^{m}$ & $3 \mathrm{~m}$ & $\mathrm{n}+1$ \\
2.11 & 1 & $2 \mathrm{~m}$ & $\mathrm{n}+2 \mathrm{~m}+1$ \\
2.12 & 1 & $2 \mathrm{~m}$ & $\mathrm{n}+1$
\end{tabular}

\subsection{The fuzzy arithmetic mean and normal arithmetic mean.}

In order to introduce the association between Fuzzy Arithmetic Mean (FAM) and Normal Arithmetic Mean(NAM), it is necessary to understand the definitions (2.1) and (2.2) which are given by Gao[2]. The first definition express a Fuzzy Number A is a triangular fuzzy number(TFN) denoted by $\left(a_{1}, a_{2}, a_{3}\right)\left(a_{1} \leq a_{2} \leq a_{3}\right)$. On the other hand, second definition explains a support of a fuzzy number $\mathrm{A}$ is an interval on a realvalued $\mathrm{R}$ denoted by

$$
\operatorname{Supp} A=\left\{\mathbf{u} \mid \mu_{A}(u)>0, u \in R\right\}
$$

if its membership function $\boldsymbol{\mu}_{\mathrm{A}}(\mathbf{u})$ is a continuous on real-valued, and $\mathbf{u}$ is called a mean value of the fuzzy number, if and only if $\boldsymbol{\mu}_{\mathrm{A}}(\mathbf{u})=\mathbf{1}$.

For example, a TFN $A=\left(a_{1}, a_{2}, a_{3}\right)\left(a_{1} \leq a_{2} \leq a_{3}\right)$, supp $A$ is an open interval $\left(\mathbf{a}_{1}, \mathbf{a}_{3}\right)$, such that, $\operatorname{supp} A=\left(\mathbf{a}_{1}, \mathbf{a}_{3}\right) \cdot \mathbf{a}_{2}$ is the mean value of this TFN.

The following two definitions on a degenerate fuzzy number(DFN) and fuzzy uniform mapping(FUM) have been supporting to clarify the relation between the fuzzy arithmetic mean and the normal arithmetic mean.

Definition 2.1.1. A fuzzy number A is called a degenerate fuzzy number denoted by $\grave{A}$, if Supp A is degenerated into point $\mathbf{a}$ on a real-valued, such that

\section{$\operatorname{Supp} A=\mathbf{a}, \quad \mu_{A}(\mathbf{a})=1,(a \in R)$.}

Especially, a TFN $\left(a_{1}, a_{2}, a_{3}\right)$ is a degenerate TFN if and only if $a_{1}=a_{2}=a_{3}$.

Definition 2.1.2. A mapping $\tilde{M}$ (x) from $\mathrm{T}(\mathrm{x})$ to a set of fuzzy numbers defined on the $\mathrm{U}$ will be called a fuzzy uniform mapping $\tilde{M}(\mathrm{x})$, if and only if the following conditions are satisfied.

(1) T(x) has $\mathbf{n}$ (n $\mathbf{E ~ N}$ ) elements, i.e., the number of values of the $X$ is $\mathbf{n}$.

(2) $\mathrm{U}$ is a bounded subset of real-valued $\mathrm{R}$, and it is divided into (n-1) equal parts.

(3) The fuzzy numbers defined on $U$ are TFNs $\left(a_{1}{ }^{i}, a_{2}{ }^{i}, a_{3}{ }^{i}\right)(i=1,2, \ldots, n)$ and $a_{1}{ }^{i}, a_{2}{ }^{i}, a_{3}{ }^{i}$ must be on points divided equally. Shapes of the TFNs are uniform isosceles triangles, and $\left(a_{1}{ }^{i+1}=a_{2}{ }^{i}=a_{3}{ }^{i-1}\right)$ is satisfied.

For example, let $\mathrm{X}$ be a linguistic variable with the label 'odd number' and with a $U=[0,10]$, then the $X$ have six values, $x_{1}=$ 'about 0 '; $x_{2}=$ 'about 2 '; $x_{3}=$ 'about 4'; $\quad \mathrm{x}_{4}=$ 'about 6 '; $\mathrm{x}_{5}=$ 'about 8 '; $\mathrm{x}_{6}=$ 'about 10 ', such that ,

$$
\mathrm{T}(\mathrm{X})=\left\{\mathrm{x}_{1}, \mathrm{X}_{2}, \mathrm{X}_{3}, \mathrm{X}_{4}, \mathrm{X}_{5}, \mathrm{X}_{6}\right\} \text { has }
$$


each value of the $\mathrm{X}$ can be described as follows;

$\mathrm{x}_{1}$ is denoted by a TFN $\mathrm{A}_{1}=(0,0,2)$

$\mathrm{x}_{2}$ is denoted by a TFN $\mathrm{A}_{2}=(0,2,4)$

$\mathrm{x}_{3}$ is denoted by a TFN $A_{3}=(2,4,6)$

$\mathrm{x}_{4}$ is denoted by a TFN $\mathrm{A}_{4}=(4,6,8)$

$\mathrm{x}_{5}$ is denoted by a TFN $\mathrm{A}_{5}=(6,8,10)$

$\mathrm{x}_{6}$ is denoted by a TFN $\mathrm{A}_{6}=(8,10,10)$.

Clearly, the mapping

$\mathrm{M}\left(\mathrm{x}_{\mathrm{i}}\right): \mathrm{T}\left(\mathrm{x}_{\mathrm{i}}\right) \rightarrow\left\{\mathrm{A}_{\mathrm{i}}\right\} \quad(\mathrm{i}=1,2, \ldots, 6) \quad$ is the Fuzzy Uniform Mapping. Based on the definitions, as an important theorem on this issue, we conclude that 'The arithmetic mean converges to the corresponding normal arithmetic mean, when $\mathrm{n}$ tends to be infinite[2].

\section{3-APPLICATION: FUZZY GOAL PROGRAMMING APPROACH TO THE FUZZY ARITHMETIC MEAN}

Up to now, the basics of fuzzy arithmetic mean and its theoretical principles have been discussed. Now, we do offer, different and new approach, called GUMAR, developed by GUNES and UMARUSMAN will be introduced.

To illustrate the step of the approach, the following simple numeric example will be used for computation of fuzzy arithmetic mean, under the assumption of normality. But in some real life evidence, it couldn't be possible to observe the existence of normality. Because of this unexpected outcome, it may be possible to redefine linguistic value to the mid value of each interval. Let the observed values be such as follows;

Interval frequencies

$2 \leq x_{1}<4$
$4 \leq x_{2}<6$
$6 \leq x_{3}<8$
$8 \leq x_{4}<10$

Fuzzy mid points of each interval of above grouped data may be considered as $\tilde{3}$, $\widetilde{5}, \widetilde{7}, \widetilde{9}$. Namely, to form all goals of the model, we could easily write the following equations as different targets; $x_{1}=\tilde{3}, x_{2}=\tilde{5}, x_{3}=\tilde{7}, x_{4}=\tilde{9}$.

It is also very easy to include other addition goals by using the computation structure of normal arithmetic mean, such as below;

$$
\text { Mean }=\frac{2 x_{1}+3 x_{2}+4 x_{3}+2 x_{4}}{11}=\frac{67}{11}=6.0909
$$


Let the definition of arithmetic mean be $\bar{X}=X_{5}=\tilde{6.09}$.

Since sum of the biases of observation from mean value is equal to zero, we do define new goal as follows;

$$
\begin{aligned}
& \sum_{i=1}^{n}\left(x_{i}-\bar{x}\right)=0 . \text { It is also possible to rewrite this equality as } \\
& \left(x_{1}-\bar{x}\right)+\left(x_{2}-\bar{x}\right)+\ldots+\left(x_{n}-\bar{x}\right)=0 \text { or } \\
& {\left[\left(x_{1}+x_{2}+\ldots+x_{n}\right)-n \bar{x}\right]=0}
\end{aligned}
$$

Now, the new goal of model could develop as below;

$$
\sum_{i=1}^{4}\left(x_{i}-\bar{x}\right)=0, \quad x_{1}+x_{2}+x_{3}+x_{4}-4 \bar{x}=0
$$

The unified view of goals of the model may summarize such as follows;

$$
\begin{aligned}
& x_{1}+x_{2}+x_{3}+x_{4}-4 \bar{x}=0 \\
& x_{1}=\tilde{3} ; x_{2}=\tilde{5} ; x_{3}=\tilde{7} ; x_{4}=\tilde{9} ; x_{5}=6.09
\end{aligned}
$$

Now we do need to determine acceptable maximum biases of each goal to model fuzzified version. Let the values of biases be $0,0.2,0.3,0.6,0.4$ and 0.5 respectively. In according to the (2.12) model, mathematical formulation of the fuzzified model of numeric example has developed, such as below;

$$
\begin{aligned}
& G_{1}: x_{1}+x_{2}+x_{3}+x_{4}-4 x_{5}=0 \ldots \ldots \ldots \ldots \mu_{1}\left(g_{1}(x)\right)=(0,0,0) \\
& G_{2}: x_{1}=\tilde{3}
\end{aligned}
$$

and the goal programming formulation of the above expressions has the following structure

$\operatorname{Max} \alpha$

$$
\begin{aligned}
& x_{1}+x_{2}+x_{3}+x_{4}-4 x_{5}=0 \\
& x_{1}+0.2 \alpha \leq 3.2 \\
& x_{2}-0.3 \alpha \geq 4.7
\end{aligned}
$$




$$
\begin{aligned}
& x_{2}+0.3 \alpha \leq 5.3 \\
& x_{3}-0.6 \alpha \geq 6.4 \\
& x_{3}+0.6 \alpha \leq 7.6 \\
& x_{4}-0.4 \alpha \geq 8.6 \\
& x_{4}+0.4 \alpha \leq 9.4 \\
& x_{5}-0.5 \alpha \geq 5.590 \\
& x_{5}+0.5 \alpha \leq 6.590 \\
& \alpha \in[0,1] \text { and } x \geq 0
\end{aligned}
$$

After this phase, it is necessary to determine acceptable maximum biases from each goal that could be symmetric or nonsymmetrical. In this application, the biases are accepted as symmetric as $0,0.2,0.3,0.6,0.4$ and 0.5 for each goal. The solution procedure has followed under the coverage of formulated model 2.12 and membership function, and then the optimal computation of fuzzy arithmetic mean has obtained, as in the following form.

$\begin{array}{lllllllll} & \mathrm{X} 1 & \mathrm{X} 2 & \mathrm{X} 3 & \mathrm{X} 4 & \mathrm{X} 5 & \mathrm{X} 6 & & \mathrm{RHS} \\ \text { Maximize } & 0 & 0 & 0 & 0 & 0 & 1 & & \\ \text { Constraint 1 } & 1 & 1 & 1 & 1 & -4 & 0 & = & 0 \\ \text { Constraint 2 } & 1 & 0 & 0 & 0 & 0 & -0,2 & >= & 2,8 \\ \text { Constraint 3 } & 1 & 0 & 0 & 0 & 0 & 0,2 & <= & 3,2 \\ \text { Constraint 4 } & 0 & 1 & 0 & 0 & 0 & -0,3 & >= & 4,7 \\ \text { Constraint 5 } & 0 & 1 & 0 & 0 & 0 & 0,3 & <= & 5,3 \\ \text { Constraint 6 } & 0 & 0 & 1 & 0 & 0 & -0,6 & >= & 6,4 \\ \text { Constraint 7 } & 0 & 0 & 1 & 0 & 0 & 0,6 & <= & 7,6 \\ \text { Constraint 8 } & 0 & 0 & 0 & 1 & 0 & -0,4 & >= & 8,6 \\ \text { Constraint 9 } & 0 & 0 & 0 & 1 & 0 & 0,4 & <= & 9,4 \\ \text { Constraint 10 } & 0 & 0 & 0 & 0 & 1 & -0,5 & >= & 5,59 \\ \text { Constraint 11 } & 0 & 0 & 0 & 0 & 1 & 0,5 & <= & 6,59\end{array}$

Values of decision variables;

$\begin{array}{ll}X_{1} & 3,020571 \\ X_{2} & 5,030858 \\ X_{3} & 7,061714 \\ X_{4} & 9,041142 \\ X_{5} & 6,038571 \text { (Mean Value) } \\ X_{6} & 0,8971426 \text { ( Membership level). }\end{array}$

List of the alternative models and their mean and membership values are listed below table. 


\begin{tabular}{cllllllll} 
& & \multicolumn{7}{c}{ Table 1 List of alternative models } \\
****** & $\mathrm{X}_{1}$ & $\mathrm{X}_{2}$ & $\mathrm{X}_{3}$ & $\mathrm{X}_{4}$ & $\mathrm{X}_{5}($ Mean $)$ & X6(Memb. Level & Bias Values \\
Model 1 & 3,020571 & 5,030858 & 7,061714 & 9,041142 & 6,038571 & 0,8971426 & Sym. \\
Model 2 & 3,028421 & 5,018947 & 7,047369 & 9,037894 & 6,033158 & 0,9052632 & Sym. \\
Model 3 & 3,022979 & 5,030638 & 7,022979 & 9,038298 & 6,028723 & 0,9234043 & Sym. \\
Model 4 & 3.050233 & 5.058605 & 7.025116 & 9.058604 & 6.048140 & 0.9162788 & Nonsy
\end{tabular}

\section{4- CONCLUSION}

If we compare the fuzzy average with fuzzy control rules and existing defuzzification operators, we find that [2].

1) The fuzzy average is not only a defuzzification method, but also a representation of fuzzy control rules by using the consequence matrix.

2) The implementation of the fuzzy average is easier than that of the fuzzy control rules. For this reason, different computation algorithm of fuzzy average has potential coverage for other effective application fields such as engineering problems with uncertainties in the parameters.

As a result; fuzzy arithmetic mean of this grouped data is 6.038571 and membership level of this value is 0.89743589 but its normal arithmetic mean value was 6.0909.In this computation, maximum acceptable bias value are;0,0.2,0.3,0.6,0.4 and 0.5, and considered as symmetric structure for each interval. In the second version of the model (Model 2), biases are also symmetric but have wider interval than previous case. This approach has increased the membership level from 0.8971426 to 0.9052632 . The third experiment on bias values has also been supporting the same objective. Further processed numeric examples showed that if it is possible to improve the membership level, value of fuzzy arithmetic mean could gradually approach to the real mean value of the gathered data from research area. The last model (Model 4), listed above, has negatively affected from nonsymmetrical biases. As a decision tool, the power of the simulation technique could exercise to develop other alternative outcomes. In brief, fuzzy goal programming approach, called GUMAR, could be used instead of the traditional computation structure, to find out the best estimation of average value of the observed data.

\section{5-REFERENCES}

1. Hasiao-Fan Wang, Ching-Chun Fu, A Generalization of Fuzzy Goal Programming With Preemptive Structure, Computers Operations Research, Vol.24,No:9,pp.819828,1997

2. Lun Shan Gao, The Fuzzy Arithmetic Mean, Fuzzy Sets and Systems 107(1999)335348

3. D. Dubois, H.Prade, Additions of Interactive Fuzzy Numbers, IEEE Trans. Automation and Control 26(1981)926-936.

4. Ni-Bin Chang, S.F. Wang, A Fuzzy Goal Programming Approach for The Optimal Planning of Metropolitan Solid Waste Management Systems, European Journal Of Operational Research 99(1997) 303-321.

5. Chih-Sheng Lee, Ching- Gung Wen, Fuzzy Goal Programming Approach for Water

Quality Management in a River Basin, Fuzzy Sets and Systems 89(1997) 181-192. 
6. M.Arenas Parra, A. Bilbao Terol, M.V.Rodriguez Uria, A Fuzzy Goal Programming Approach to Portfolio Selection, European Journal of Operational Research 133,(2001) 287-297.

7. Liang-Hsuan Chen, Feng-ChouTsai, Fuzzy Goal Programming with Different Importance and Priorities, Theory and Methodology, European Journal of Operational Research 133(2001) 548-556.

8. T. K. Roy and M. Maiti, Multi-Objective Inventory Models of Deteriorating Items With Some Constraints in a Fuzzy Environment, Computer Operations Research, Vol.25, No.12, pp.1085, 1095,1998

9. Jong Soon Kim, Kyu-Seung Whang, The Tolerance Approach to the Fuzzy Goal Programming Problems with Unbalanced Triangular Membership Function, European Journal of Operational Research 107(1998) 614-624.

10. R.Narasimhan, Goal Programming in Fuzzy Environment, Decision Sciences 11(1980) 325-336

11. Young-Jou Lai,Ching-L. Hwang, Fuzzy Multiple Objective Decision Making, Springer-Verlag New York, 1994.

12. Liang-Hsuan Chen, Feng-Chou Tsai, Fuzzy Goal Programming With Different Importance and Priorities, European Journal of Operational Research 133(20010 548556. 